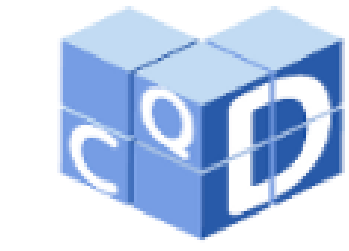

Revista Eletrônica

Paulista de Matemática

ISSN 2316-9664

Volume 18, jul. 2020

\footnotetext{
Allan Kenedy Santos Silva

Instituto de Matemática

Universidade Federal de Alagoas

allan.santos@im.ufal.br
}

\section{Uma prova do Teorema de Green}

A proof of the Green's theorem

\section{Resumo}

Na maioria dos livros de cálculo o Teorema de Green é enunciado para regiões com fronteira $C^{1}$ por partes, porém, é demonstrado apenas um caso especial em que a região de integração é uma região simples ou que pode ser decomposta numa reunião finita de regiões simples. Em vários desses livros é afirmado que o teorema vale para regiões mais gerais, mas não o demonstram. O objetivo deste texto é provar o Teorema de Green para o caso geral enunciado.

Palavras-chave: Teorema de Green. Integral de linha. Integral múltipla. Linha poligonal.

\section{Abstract}

In most calculus books Green's theorem is stated for $C^{1}$ boundary regions by parts, but only one special case is shown where the integration region is a simple region or can be decomposed into a finite meeting of simple regions. In several of these books it is stated that the theorem holds for more general regions but does not proof it. The purpose of this paper is to proof Green's Theorem for the general case stated.

Keywords: Green's theorem. Line integral. Multiple integral. Polygonal line. 


\section{Introdução}

"Sejam $D$ um compacto com fronteira $\operatorname{fr}(D)$ seccionalmente suave contida num aberto $U \subset \mathbb{R}^{2}$ e $P, Q: U \rightarrow \mathbb{R}$ funções de classe $C^{1}$. Então

$$
\int_{f r(D)} P d x+Q d y=\iint_{D}\left(\frac{\partial Q}{\partial x}-\frac{\partial P}{\partial y}\right) d x d y
$$

com a integração sendo feita no sentido anti-horário."

Este é um enunciado típico dos livros de cálculo. Contudo, nesses livros o Teorema de Green é demonstrado apenas para um caso especial em que a região de integração é uma região simples (dos tipos 1 e 2 simultaneamente conforme as definições 2.1 e 2.2 a seguir) ou pode ser decomposta numa reunião finita de regiões simples (COLLEY, 2012; COURANT, 1936; GONÇALVES; FLEMMING, 2007; LEITHOLD, 1994; PISKUNOV, 1997; STEWART, 2009; THOMAS et al, 2009;). Outras referências afirmam que uma região que cumpre as condições do enunciado acima pode ser decomposta numa reunião finita de regiões dos tipos 1 ou 2, mas não provam essa afirmação (LANCELOTTI, 201-?; LINS NETO, 2008). Buscando referências bibliográficas presentes sobre cálculo avançado, foi observado que o Teorema de Green é demonstrado como caso particular de uma versão generalizada do Teorema de Stokes (LIMA, 2015; MUNKRES, 1991; SPIVAK, 1965). Contudo, para o seu entendimento são necessários conhecimentos de cálculo vetorial avançado (formas diferenciais, variedades, diferencial exterior dentre outros tópicos).

Uma abordagem mais elementar consiste em aproximar o cálculo da integral ao longo de uma região (e sua fronteira) por meio de polígonos, os quais vale o teorema (ver lema 3.1). Tal estratégia é apresentada em Guerra (2010), porém existem diversas afirmações feitas sem demonstração e algumas passagens obscuras.

Outra abordagem vista foi apresentar o Teorema de Green como caso particular do chamado Teorema de Kelvin, o qual mostra como a integral de um campo vetorial não conservativo varia ao longo de caminhos homotópicos; contudo, toma hipóteses mais restritivas para simplificar as contas (por exemplo, que a homotopia entre dois caminhos é de classe $C^{2}$ ). Os detalhes podem ser vistos em Acker (2011).

O objetivo deste texto é demonstrar o Teorema de Green para o caso geral enunciado sem recorrer a formulações avançadas. Para tal será adotada a seguinte estratégia: será mostrado inicialmente que o teorema vale para regiões limitadas por linhas poligonais; em seguida, que integrais sobre regiões e integrais curvilíneas podem ser calculadas de forma aproximada por meio de integrais sobre linhas ou regiões poligonais; com isso, efetuando tais aproximações se demonstrará o caso geral.

\section{Teorema de Green para regiões simples}

Começaremos mostrando a validade do Teorema de Green para regiões simples, como feito nos livros de cálculo.

Definição 2.1. Um conjunto $D \subset \mathbb{R}^{2}$ é uma região do tipo 1 se existem funções $\alpha, \beta:[a, b] \rightarrow \mathbb{R}$ de classe $C^{1}$ por partes tais que $\alpha(x) \leq \beta(x) \forall x \in[a, b]$ e $D=$ $\left\{(x, y) \in \mathbb{R}^{2} \mid a \leq x \leq b, \alpha(x) \leq y \leq \beta(x)\right\}$.

Definição 2.2. Um conjunto $D \subset \mathbb{R}^{2}$ é uma região do tipo 2 se existem funções $\alpha, \beta:[a, b] \rightarrow \mathbb{R}$ de classe $C^{1}$ por partes tais que $\alpha(x) \leq \beta(x) \forall x \in[a, b]$ e $D=$ $\left\{(x, y) \in \mathbb{R}^{2} \mid a \leq y \leq b, \alpha(y) \leq x \leq \beta(y)\right\}$. 
Figura 1

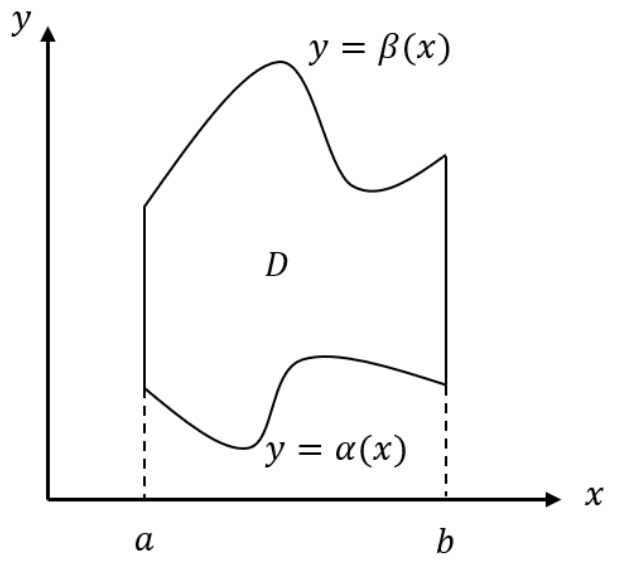

(a) Região do tipo 1 .

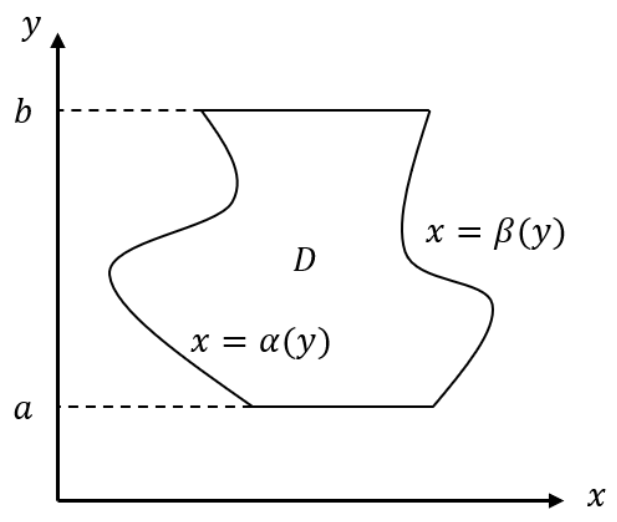

(b) Região do tipo 2

Fonte: $\mathrm{O}$ autor.

Lema 2.3 (Teorema de Green para regiões simples). Se $D$ é uma região do tipo 1 ou 2 e $P, Q: \bar{D} \rightarrow \mathbb{R}$ são de classe $C^{1}$, então

$$
\int_{f r(D)} P d x+Q d y=\iint_{D}\left(\frac{\partial Q}{\partial x}-\frac{\partial P}{\partial y}\right) d x d y
$$

com a integração sendo feita no sentido anti-horário. ${ }^{1}$

Prova: Ver Lins Neto (2008, p. 175-177) e Lancelotti (201-?, p. 1-4).

\section{Teorema de Green para polígonos}

Lema 3.1 (Teorema de Green para polígonos). Seja $D \subset \mathbb{R}^{2}$ uma região limitada cuja fronteira é uma linha poligonal simples. Então para quaisquer $P, Q: \bar{D} \rightarrow \mathbb{R}$ de classe $C^{1}$ :

$$
\int_{f r(D)} P d x+Q d y=\iint_{D}\left(\frac{\partial Q}{\partial x}-\frac{\partial P}{\partial y}\right) d x d y
$$

com a integração sendo feita no sentido anti-horário.

Prova: De acordo com Lima (1991), um polígono de $n$ lados pode ser decomposto em $n-2$ triângulos por meio de $n-3$ diagonais contidas no interior do polígono. Assim tem-se a decomposição

$$
D=\bigcup_{i=1}^{n-2} T_{i}
$$

em que $\forall i \neq j, \operatorname{int}\left(T_{i}\right) \cap \operatorname{int}\left(T_{j}\right)=\emptyset$ e $f r\left(T_{i}\right) \cap f r\left(T_{j}\right)=\varnothing$ ou um vértice ou um lado.

${ }^{1}$ A rigor, o correto é dizer que a integral da forma diferencial $P d x+Q d y$ é feita ao longo do bordo de $D$ $(\partial D)$, uma vez que a fronteira $f r(D)$ é a imagem de uma parametrização desse bordo, mas acredito que o leitor não terá problemas com essa mistura de termos. 
Figura 2: Decomposição da região $D$.

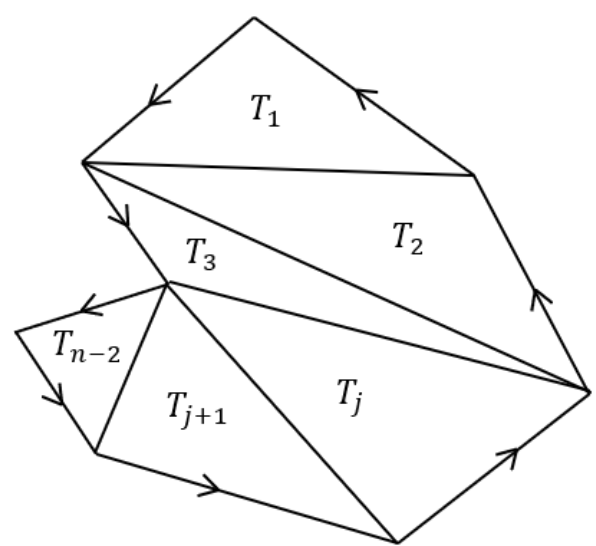

Fonte: $\mathrm{O}$ autor.

Observe que

$$
\int_{f r(D)} P d x+Q d y=\int_{f r\left(\cup_{i=1}^{n-2} T_{i}\right)} P d x+Q d y=\sum_{i=1}^{n-2} \int_{f r\left(T_{i}\right)} P d x+Q d y
$$

De fato, se os triângulos $T_{i}$ e $T_{j}$ possuem um lado $L$ em comum, denotando $T_{i k}$ como o lado $k$ do triângulo $T_{i}$ temos (ver Figura 3)

$$
\begin{gathered}
\int_{f r\left(T_{i}\right)} P d x+Q d y+\int_{f r\left(T_{j}\right)} P d x+Q d y= \\
\int_{T_{i 1}} P d x+Q d y+\int_{T_{i 2}} P d x+Q d y+\int_{L} P d x+Q d y+\int_{T_{j 1}} P d x+Q d y+\int_{T_{j 2}} P d x+Q d y \\
+\int_{-L} P d x+Q d y
\end{gathered}
$$

pois o lado $L$ tem orientações opostas ${ }^{2}$ nos triângulos $T_{i}$ e $T_{j}$ e assim as integrais ao longo de $L$ se cancelam, e a soma das integrais restantes é igual a integral ao longo da fronteira de $T_{i} \cup$ $T_{j}$, isto é,

$$
\int_{f r\left(T_{i}\right)} P d x+Q d y+\int_{f r\left(T_{j}\right)} P d x+Q d y=\int_{f r\left(T_{i} \cup T_{j}\right)} P d x+Q d y
$$

Figura 3: Regiões de integração $T_{i}$ e $T_{j}$.

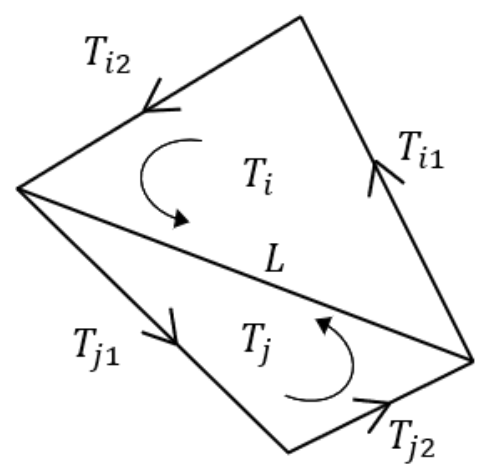

Fonte: $\mathrm{O}$ autor.

\footnotetext{
${ }^{2} \mathrm{Um}$ argumento mais rigoroso para este fato pode ser encontrado em Lins Neto (2008, p. 178-179).
} 
No caso de $\operatorname{fr}\left(T_{i}\right) \cap f r\left(T_{j}\right)$ ser vazio ou um vértice, a igualdade (3) é trivialmente satisfeita. Efetuando o raciocínio acima para todos os triângulos da decomposição, observando que as integrais sobre as diagonais se cancelam, chegamos à igualdade (2).

Como um triângulo é claramente uma região do tipo 1 ou 2, segue do lema 2.3 que

e como

$$
\int_{f r\left(T_{i}\right)} P d x+Q d y=\iint_{T_{i}}\left(\frac{\partial Q}{\partial x}-\frac{\partial P}{\partial y}\right) d x d y
$$

obtém-se então a igualdade (1).

$$
\iint_{D}\left(\frac{\partial Q}{\partial x}-\frac{\partial P}{\partial y}\right) d x d y=\sum_{i=1}^{n-2} \iint_{T_{i}}\left(\frac{\partial Q}{\partial x}-\frac{\partial P}{\partial y}\right) d x d y
$$

\section{Teorema de Green geral}

Iniciamos esta seção relembrando algumas definições e proposições sobre funções contínuas em conjuntos compactos.

Definição 4.1. Uma função $f: X \subset \mathbb{R}^{m} \rightarrow \mathbb{R}^{n}$ é uniformemente contínua se para cada $\varepsilon>0$, existe $\delta>0$ tal que para quaisquer $x, y \in X,|x-y|<\delta \Rightarrow|f(x)-f(y)|<\varepsilon$.

Proposição 4.2. Toda aplicação contínua $f: X \rightarrow \mathbb{R}^{n}$, definida num conjunto compacto $X \subset \mathbb{R}^{m}$, é uniformemente contínua.

Prova: Ver Lima (2015, p. 47), teorema 21

Proposição 4.3. Se $f: X \rightarrow \mathbb{R}^{n}$ é contínua num compacto $X \subset \mathbb{R}^{m}$, então $f(X)$ é compacto.

Prova: Ver Lima (2015, p. 45), teorema 20

Corolário 4.4 (Weierstrass). Se $f: X \rightarrow \mathbb{R}$ é contínua num compacto $X \subset \mathbb{R}^{m}$, então atinge seus valores máximo e mínimo em $X$.

Definição 4.5. Um caminho $f:[a, b] \rightarrow \mathbb{R}^{n}$ é uniformemente diferenciável se dado $\varepsilon>0$, existe $\delta>0$ tal que

$$
x, x+h \in[a, b], 0<|h|<\delta \Rightarrow\left|f(x+h)-f(x)-h f^{\prime}(x)\right|<\varepsilon|h|
$$

ou de forma equivalente, se existe $\delta>0$ tal que

ou

$$
x, y \in[a, b], 0<|x-y|<\delta \Rightarrow\left|\frac{f(x)-f(y)}{x-y}-f^{\prime}(x)\right|<\varepsilon
$$

$$
x, y \in[a, b], 0<|x-y|<\delta \Rightarrow\left|\frac{f(x)-f(y)}{x-y}-f^{\prime}(y)\right|<\varepsilon
$$

Proposição 4.6. Um caminho $f:[a, b] \rightarrow \mathbb{R}^{n}$ é uniformemente diferenciável se, e somente se é de classe $C^{1}$. 89).

Prova: Para $n=1$ veja Lima (2016, p. 277), teorema 8, e para $n \geq 2$ veja Lima (2015, p.

Os lemas seguintes mostram que o cálculo de uma integral de linha pode ser aproximado pelo cálculo da integral sobre uma linha poligonal.

Lema 4.7. Seja $\gamma:[a, b] \rightarrow \mathbb{R}^{n}$ uma curva $C^{1}$ por partes. Dado $\varepsilon>0$, existe uma partição $\mathcal{P}=\left\{t_{0}=a<t_{1}<\cdots<t_{m}=b\right\}$ tal que para quaisquer $i=1, \ldots, m$,

$$
\xi, \zeta \in\left[t_{i-1}, t_{i}\right] \Rightarrow\left|\gamma(\xi)-\eta_{i}(\zeta)\right|<\varepsilon e\left|\gamma^{\prime}(\xi)-\eta_{i}^{\prime}(\zeta)\right|<\varepsilon
$$

em que $\eta_{i}$ é o segmento de reta de extremidades $\gamma\left(t_{i-1}\right)$ e $\gamma\left(t_{i}\right)$. 
Prova: Começamos com o caso $\gamma$ ser de classe $C^{1}$ simplesmente. $\gamma$ e $\gamma^{\prime}$ são contínuas no compacto $[a, b], \operatorname{logo}$ são uniformemente contínuas. Dessa forma, dado $\varepsilon>0$, existem $\delta_{1}>$ 0 e $\delta_{2}>0$ tais que para quaisquer $s, t \in[a, b]$

$\mathrm{e}$

$$
|s-t|<\delta_{1} \Rightarrow|\gamma(s)-\gamma(t)|<\frac{\varepsilon}{2}
$$

$$
|s-t|<\delta_{2} \Rightarrow\left|\gamma^{\prime}(s)-\gamma^{\prime}(t)\right|<\frac{\varepsilon}{2}
$$

Pela proposição 4.6, $\gamma$ é uniformemente diferenciável, logo existe $\delta_{3}>0$ tal que

$$
|s-t|<\delta_{3} \Rightarrow\left|\frac{\gamma(s)-\gamma(t)}{s-t}-\gamma^{\prime}(t)\right|<\frac{\varepsilon}{2}
$$

Tome uma partição $\mathcal{P}=\left\{t_{0}=a<t_{1}<\cdots<t_{m}=b\right\}$ tal que $|\mathcal{P}|<\min \left\{\delta_{1}, \delta_{2}, \delta_{3}\right\}$, onde $|\mathcal{P}|=\max _{1 \leq i \leq m}\left(t_{i}-t_{i-1}\right)$. Defina, para cada $i=1, \ldots, m$,

$$
\eta_{i}(t)=\gamma\left(t_{i-1}\right)+\frac{t-t_{i-1}}{t_{i}-t_{i-1}}\left(\gamma\left(t_{i}\right)-\gamma\left(t_{i-1}\right)\right), t \in\left[t_{i-1}, t_{i}\right]
$$

Teremos para quaisquer $\xi, \zeta \in\left[t_{i-1}, t_{i}\right]$ :

$$
\left|\gamma(\xi)-\eta_{i}(\zeta)\right| \leq\left|\gamma\left(t_{i-1}\right)-\gamma(\xi)\right|+\left|\gamma\left(t_{i}\right)-\gamma\left(t_{i-1}\right)\right|<\varepsilon
$$

$\mathrm{e}$

$$
\left|\gamma^{\prime}(\xi)-\eta_{i}^{\prime}(\zeta)\right| \leq\left|\frac{\gamma\left(t_{i}\right)-\gamma\left(t_{i-1}\right)}{t_{i}-t_{i-1}}-\gamma^{\prime}\left(t_{i-1}\right)\right|+\left|\gamma^{\prime}\left(t_{i-1}\right)-\gamma^{\prime}(\xi)\right|<\varepsilon
$$

o que finaliza a prova para este caso. Se $\gamma$ é $C^{1}$ por partes, tome uma partição $\mathcal{P}^{\prime}=$ $\left\{t_{0}^{\prime}=a<t_{1}^{\prime}<\cdots<t_{m^{\prime}}^{\prime}=b\right\}$ tal que $\gamma$ é $C^{1}$ em cada $\left[t_{i-1}^{\prime}, t_{i}^{\prime}\right], i=1, \ldots, m^{\prime}$. Dado $\varepsilon>0$, temos em cada $\left[t_{i-1}^{\prime}, t_{i}^{\prime}\right]$ uma partição $\mathcal{P}_{i}^{\prime}=\left\{t_{i, 0}=t_{i-1}^{\prime}<t_{i, 1}<\cdots<t_{i, j}=t_{i}^{\prime}\right\}$ tal que para cada $k=1, \ldots, j$,

$$
\xi, \zeta \in\left[t_{i, k-1}, t_{i, k}\right] \Rightarrow\left|\gamma(\xi)-\eta_{i, k}(\zeta)\right|<\varepsilon \text { e }\left|\gamma^{\prime}(\xi)-\eta_{i, k}^{\prime}(\zeta)\right|<\varepsilon
$$

em virtude do primeiro caso. Tomando a partição $\mathcal{P}=\bigcup_{i=1}^{m^{\prime}} \mathcal{P}_{i}^{\prime}=\left\{t_{0}=a<t_{1}<\cdots<t_{m}=\right.$ b\} teremos então

para cada $i=1, \ldots, m$.

$$
\xi, \zeta \in\left[t_{i-1}, t_{i}\right] \Rightarrow\left|\gamma(\xi)-\eta_{i}(\zeta)\right|<\varepsilon \text { e }\left|\gamma^{\prime}(\xi)-\eta_{i}^{\prime}(\zeta)\right|<\varepsilon
$$

Observação 1: Perceba pela demonstração do lema que se uma partição $\mathcal{P}^{\prime}$ refina $\mathcal{P}$, as desigualdades do lema valem também para $\mathcal{P}^{\prime}$, pois $\left|\mathcal{P}^{\prime}\right| \leq|\mathcal{P}|$.

Lema 4.8. Sejam $\gamma:[a, b] \rightarrow \mathbb{R}^{n}$ uma curva $C^{1}$ por partes cujo traço $\{\gamma\}=\gamma([a, b])$ está contido num aberto $U \subset \mathbb{R}^{n}$ e $F: U \rightarrow \mathbb{R}^{n}$ um campo vetorial contínuo. Dado $\varepsilon>0$, existe uma linha poligonal $P \subset U$ cujos vértices pertencem a $\{\gamma\}$ tal que

$$
\left|\int_{\gamma} F-\int_{P} F\right|<\varepsilon
$$

Prova: Por ser $C^{1}$ por partes, $\gamma$ pode ser parametrizada pelo comprimento de arco. Com isso podemos supor, sem perda de generalidade, que $\gamma:[0, L] \rightarrow \mathbb{R}^{n}$, onde $L$ é o comprimento de $\gamma$.

Fixemos um $r$ tal que $0<r<d=d(\{\gamma\}, f r(U))$, em que $d(\{\gamma\}, f r(U))=$ $\inf \{|\gamma(t)-u| ; t \in[0, L], u \in f r(U)\}$ é a distância do conjunto $\{\gamma\}$ ao conjunto $f r(U)$. Se $f r(U)=\emptyset$, tome um $r>0$ qualquer. ${ }^{3}$ Desse modo, $\{\gamma\}$ está contido no compacto $V=$ $\bigcup_{t \in[0, L]} B[\gamma(t) ; r] \subset U$, em que $B[\gamma(t) ; r]=\left\{u \in \mathbb{R}^{n} ;|u-\gamma(t)| \leq r\right\}$ é a bola fechada de centro $\gamma(t)$ e raio $r$.

\footnotetext{
${ }^{3}$ Note que $d \neq 0$, pois do contrário existiria um ponto $u \in f r(U)$ tal que $u \in\{\gamma\} \subset U$, contradizendo a hipótese de $U$ ser aberto. Note que se $f(U)=\emptyset$, então $U=\mathbb{R}^{n}$.
} 
Figura 4

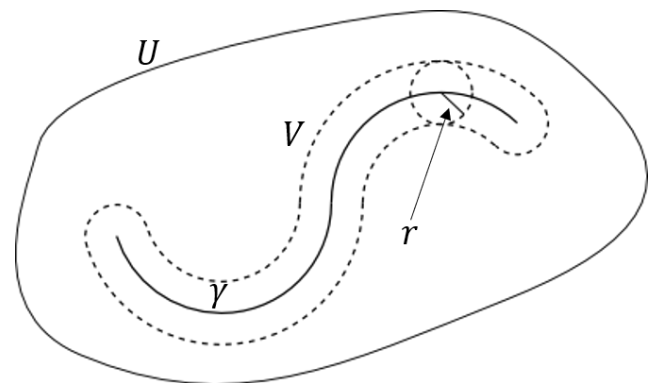

(a) Cobertura da curva $\gamma$.

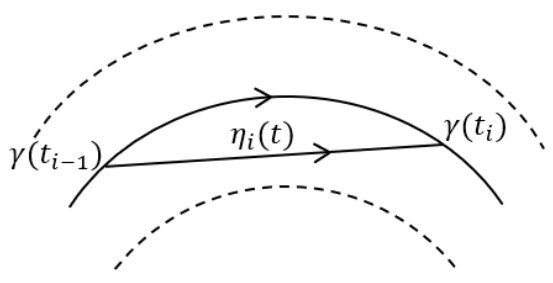

(b) Um lado da linha poligonal.

Fonte: $\mathrm{O}$ autor.

$F$ é contínua em $V$, logo é uniformemente contínua nele em virtude da proposição 4.2. Portanto, fixado $\varepsilon>0$, existe $\delta>0$ tal que $\forall u, v \in V,|u-v|<\delta \Rightarrow|F(u)-F(v)|<$ $\varepsilon / 2 N L$, onde $N=\max _{t \in[0, L]}\left|\gamma^{\prime}(t)\right|$, cuja existência é garantida pelo corolário 4.4.

Pelo lema 4.7, existe uma partição $\mathcal{P}=\left\{t_{0}=0<t_{1}<\cdots<t_{m}=L\right\}$ tal que para quaisquer $\xi, \zeta \in\left[t_{i-1}, t_{i}\right]$ se tem

$$
\left|\gamma(\xi)-\eta_{i}(\zeta)\right|<\bar{\varepsilon} \text { e }\left|\gamma^{\prime}(\xi)-\eta_{i}^{\prime}(\zeta)\right|<\bar{\varepsilon}
$$

onde $\bar{\varepsilon}=\min \left\{r, \delta, \frac{\varepsilon}{2 M L}\right\}$ e $M=\max _{x \in V}|F(x)|$ (corolário 4.4). Como $\left|\gamma(\xi)-\eta_{i}(\zeta)\right|<\delta$ temos

$$
\left|F(\gamma(\xi))-F\left(\eta_{i}(\zeta)\right)\right|<\frac{\varepsilon}{2 N L}
$$

Indicando por $\gamma_{i}$ a restrição de $\gamma$ ao intervalo $\left[t_{i-1}, t_{i}\right]$, existem, pelo Teorema do Valor Médio para integrais ${ }^{4}, \xi_{i}, \zeta_{i} \in\left(t_{i-1}, t_{i}\right)$ tais que

$$
\begin{aligned}
& \int_{\gamma_{i}} F=\int_{t_{i-1}}^{t_{i}}\left\langle F(\gamma(t)), \gamma^{\prime}(t)\right\rangle d t=\left\langle F\left(\gamma\left(\xi_{i}\right)\right), \gamma^{\prime}\left(\xi_{i}\right)\right\rangle\left(\mathrm{t}_{\mathrm{i}}-t_{i-1}\right) \\
& \int_{\eta_{i}} F=\int_{t_{i-1}}^{t_{i}}\left\langle F\left(\eta_{i}(t)\right), \eta_{i}^{\prime}(t)\right\rangle d t=\left\langle F\left(\eta_{i}\left(\zeta_{i}\right)\right), \eta_{i}^{\prime}\left(\zeta_{i}\right)\right\rangle\left(\mathrm{t}_{\mathrm{i}}-t_{i-1}\right)
\end{aligned}
$$

para cada $i=1, \ldots, m$. Dessa forma

$$
\begin{aligned}
\left|\int_{\gamma_{i}} F-\int_{\eta_{i}} F\right| & =\left|\left\langle F\left(\gamma\left(\xi_{i}\right)\right), \gamma^{\prime}\left(\xi_{i}\right)\right\rangle-\left\langle F\left(\eta_{i}\left(\zeta_{i}\right)\right), \eta_{i}^{\prime}\left(\zeta_{i}\right)\right\rangle\right|\left(\mathrm{t}_{\mathrm{i}}-t_{i-1}\right) \\
& \leq\left[\left|F\left(\gamma\left(\xi_{i}\right)\right)-F\left(\eta_{i}\left(\zeta_{i}\right)\right)\right|\left|\gamma^{\prime}\left(\xi_{i}\right)\right|+\left|F\left(\eta_{i}\left(\zeta_{i}\right)\right)\right|\left|\gamma^{\prime}\left(\xi_{i}\right)-\eta_{i}^{\prime}\left(\zeta_{i}\right)\right|\right]\left(\mathrm{t}_{\mathrm{i}}-t_{i-1}\right) \\
& <\left(\frac{\varepsilon}{2 N L} \cdot N+\frac{\varepsilon}{2 M L} \cdot M\right)\left(\mathrm{t}_{\mathrm{i}}-t_{i-1}\right)=\frac{\varepsilon}{L}\left(\mathrm{t}_{\mathrm{i}}-t_{i-1}\right)
\end{aligned}
$$

Chamando $P$ a justaposição dos caminhos $\eta_{i}, i=1, \ldots, m$, temos finalmente

$$
\left|\int_{\gamma} F-\int_{P} F\right| \leq \sum_{i=1}^{m}\left|\int_{\gamma_{i}} F-\int_{\eta_{i}} F\right|<\sum_{i=1}^{m} \frac{\varepsilon}{L}\left(\mathrm{t}_{\mathrm{i}}-t_{i-1}\right)=\frac{\varepsilon}{L} \cdot L=\varepsilon
$$

Observação 2: Note que $P$ é obtida de uma partição $\mathcal{P}$ de $[a, b]$. Se $\mathcal{P}^{\prime}$ refina $\mathcal{P}$ e $P^{\prime}$ é a linha poligonal obtida de $\mathcal{P}^{\prime}$, então a desigualdade do lema vale também para $P^{\prime}$. Isso decorre da observação 1.

Vamos discutir brevemente o cálculo da área de uma região plana.

Seja $D$ um conjunto limitado contido num retângulo $R$. A área interna da região $D$ e a área externa dela são definidas respectivamente como

\footnotetext{
${ }^{4}$ Se $f:[a, b] \rightarrow \mathbb{R}$ é contínua, então existe $c \in(a, b)$ tal que $\int_{a}^{b} f(x) d x=f(c)(b-a)$ (demonstração em Lima (2016, p. 327), teorema 12).
} 


$$
\begin{aligned}
& \text { a.int. }(D)=\sup _{\mathcal{P}} \sum_{R_{i} \in \mathcal{P}} \inf _{(x, y) \in R_{i}} \xi(x, y) \cdot \text { a. }\left(R_{i}\right)=\frac{\underline{\underline{\int}} \xi(x, y) d x d y}{R} \\
& \text { a.ext. }(D)=\inf _{\mathcal{P}} \sum_{R_{i} \in \mathcal{P}} \sup _{(x, y) \in R_{i}} \xi(x, y) \cdot \text { a. }\left(R_{i}\right)=\overline{\iint_{R}} \xi(x, y) d x d y
\end{aligned}
$$

onde $\mathcal{P}$ é uma partição do retângulo $R$ em sub-retângulos $R_{i}$, a. $\left(R_{i}\right)$ é a área de $R_{i}$ e $\xi: \mathbb{R}^{2} \rightarrow$ $\mathbb{R}$ definida por

é a função característica de $D$.

$$
\xi(x, y)=\left\{\begin{array}{l}
1 \text { se }(x, y) \in D \\
0 \text { se }(x, y) \notin D
\end{array}\right.
$$

a. int. $(D)$ é interpretada como o limite da soma das áreas dos retângulos contidos no interior de $D$ (aproximação por falta) e a. ext. $(D)$ é o limite da soma das áreas dos retângulos que contém pontos de $D$ (aproximação por excesso). ${ }^{5}$

Figura 5: Somas parciais para a área da região.

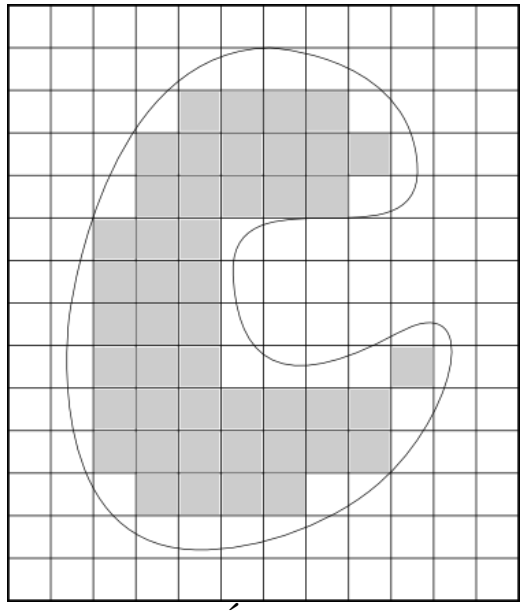

(a) Área interna.

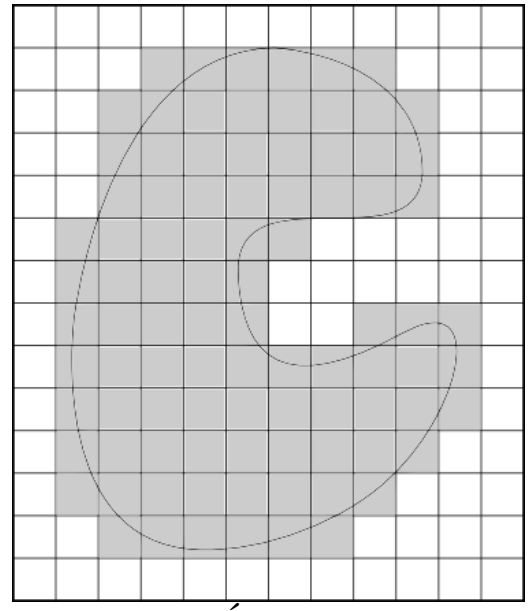

(b) Área externa.

Fonte: $\mathrm{O}$ autor.

O conjunto $D$ é dito $J$-mensurável se a. int. $(D)=$ a. ext. $(D)$ e esse valor é definido como a área de $D$ :

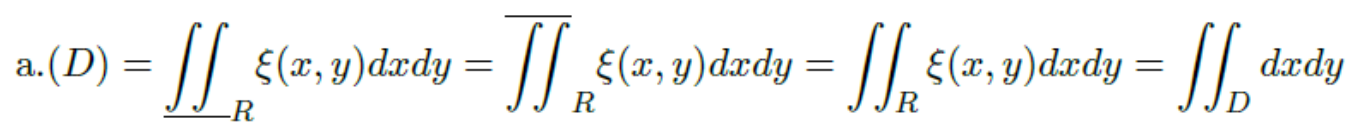

Isto significa que, dado $\varepsilon>0$, existe uma partição $\mathcal{P}$ tal que

$$
\sum_{R_{i} \in \mathcal{P}} \sup _{(x, y) \in R_{i}} \xi(x, y) \cdot \text { a. }\left(R_{i}\right)-\sum_{R_{i} \in \mathcal{P}} \inf _{(x, y) \in R_{i}} \xi(x, y) \cdot \text { a. }\left(R_{i}\right)<\varepsilon
$$

e esta diferença é a soma das áreas dos retângulos que contêm pontos de $\operatorname{fr}(D)$. Refinando esta partição (isto é, tomando uma partição $\mathcal{Q} \supset \mathcal{P}$ ), a desigualdade acima continua válida.

De acordo com Lima (2015, p. 364), teorema 5, um conjunto limitado $D$ é J-mensurável se, e somente se sua fronteira tem medida nula (med. $f r(D)=0$ ), isto é, se, dado $\varepsilon>0$, existe uma cobertura enumerável de blocos abertos $\cup C_{i} \supset \operatorname{fr}(D)$ tal que $\sum$ a. $\left(C_{i}\right)<\varepsilon$. Se $\operatorname{fr}(D)$ é um caminho $C^{1}$, então med. $f r(D)=0$ (LIMA, 2015, p. 357). Se $f r(D)$ é $C^{1}$ por partes, cada parte sua onde é $C^{1}$ tem medida nula, e como uma união enumerável de conjuntos de medida nula tem medida nula, temos que $f r(D)$ tem medida nula (LIMA, 2015, p. 352). Toda

\footnotetext{
${ }^{5} \mathrm{Na}$ verdade, seria o supremo e o ínfimo das somas das áreas dos retângulos sobre todas as partições possíveis de $R$, mas a expressão "limite“ é matematicamente válida conforme Lima (2015, p. 373), teorema 10.
} 
essa discussão serve para mostrar que a integração sobre $D$ cuja fronteira é $C^{1}$ por partes tem sentido.

Estamos finalmente em condições de demonstrar o

Teorema 4.9 (Teorema de Green). Sejam $D \subset \mathbb{R}^{2}$ um conjunto limitado simplesmente conexo com fronteira $f r(D)$ parametrizada por uma curva $\gamma:[a, b] \rightarrow \mathbb{R}^{2}$ simples fechada $C^{1}$ por partes, $U \subset \mathbb{R}^{2}$ um aberto contendo $\bar{D}$ e $P, Q: U \rightarrow \mathbb{R}$ de classe $C^{1}$. Então

$$
\int_{\gamma} P d x+Q d y=\iint_{D}\left(\frac{\partial Q}{\partial x}-\frac{\partial P}{\partial y}\right) d x d y
$$

com a integração sendo feita no sentido anti-horário.

Prova: $\mathrm{O}$ argumento a seguir será baseado na seguinte proposição: se $x, y$ são números reais com a propriedade que para todo $\varepsilon>0$ vale $|x-y|<\varepsilon$, então $x=y \cdot{ }^{6}$ Ora, dado $\varepsilon>0$, assuma, por enquanto, que existe uma linha poligonal $P$ - não confundir com a função $P$ limitando uma região poligonal $\Pi$ tal que

$$
\left|\int_{\gamma} \mathrm{Pdx}+\mathrm{Qdy}-\int_{\mathrm{P}} \mathrm{Pdx}+\mathrm{Qdy}\right|<\frac{\varepsilon}{2}
$$

$\mathrm{e}$

Como

$$
\left|\iint_{D}\left(\frac{\partial Q}{\partial x}-\frac{\partial P}{\partial y}\right) d x d y-\iint_{\Pi}\left(\frac{\partial Q}{\partial x}-\frac{\partial P}{\partial y}\right) d x d y\right|<\frac{\varepsilon}{2}
$$

devido ao lema 3.1 temos pela desigualdade triangular que

$$
\int_{\mathrm{P}} P d x+Q d y=\iint_{\Pi}\left(\frac{\partial Q}{\partial x}-\frac{\partial P}{\partial y}\right) d x d y
$$

$$
\begin{gathered}
\left|\iint_{D}\left(\frac{\partial Q}{\partial x}-\frac{\partial P}{\partial y}\right) d x d y-\int_{\gamma} P d x+Q d y\right| \leq \\
\left|\iint_{D}\left(\frac{\partial Q}{\partial x}-\frac{\partial P}{\partial y}\right) d x d y-\iint_{\Pi}\left(\frac{\partial Q}{\partial x}-\frac{\partial P}{\partial y}\right) d x d y\right|+\left|\int_{\gamma} P d x+Q d y-\int_{P} P d x+Q d y\right|< \\
\frac{\varepsilon}{2}+\frac{\varepsilon}{2}=\varepsilon
\end{gathered}
$$

logo, pela proposição inicial

$$
\int_{\gamma} P d x+Q d y=\iint_{D}\left(\frac{\partial Q}{\partial x}-\frac{\partial P}{\partial y}\right) d x d y
$$

o que demonstra o teorema. Resta agora mostrar a existência da tal linha poligonal $P$.

Fixamos um $\varepsilon>0$. Pelo lema 4.8 existe uma linha poligonal $P^{\prime}$ orientada no mesmo sentido que $\gamma$ e esta linha está contida num compacto $V \subset U$ (o conjunto construído no lema 4.8) tal que

$$
\left|\int_{\gamma} P d x+Q d y-\int_{P^{\prime}} P d x+Q d y\right|<\frac{\varepsilon}{2}
$$

Tal linha poligonal produz uma partição $\mathcal{P}^{\prime}=\left\{t_{0}^{\prime}=a<t_{1}^{\prime}<\cdots<t_{n^{\prime}}^{\prime}=b\right\}$ tal que os vértices de $P^{\prime}$ são da forma $\gamma\left(t_{i}^{\prime}\right)$. Para cada $i=0, \ldots, n^{\prime}$, traçamos retas paralelas aos eixos $x$ e $y$ passando pelos pontos $\gamma\left(t_{i}^{\prime}\right)$. Tais retas formam uma partição $\mathcal{Q}$ de um retângulo $R \supset V$ (Figura 6a). Obviamente $R \supset\{\gamma\}$. Pela discussão anterior sobre áreas, existe uma partição $\mathcal{R}$ de $R$ tal que

$$
\text { soma das áreas dos retângulos que contêm pontos de } \operatorname{fr}(D)<\frac{\varepsilon}{2 M}
$$

\footnotetext{
${ }^{6}$ Isto é mais fácil provar do que enunciar: Se $x \neq y$, tome $\varepsilon=|x-y|$ e obtenha o absurdo que $|x-y|<$ $\varepsilon=|x-y|$.
} 
em que

Tomamos a partição $\mathcal{Q} \cup \mathcal{R}$.

$$
M=\max _{V \cup D}\left|\frac{\partial Q}{\partial x}-\frac{\partial P}{\partial y}\right|
$$

Figura 6

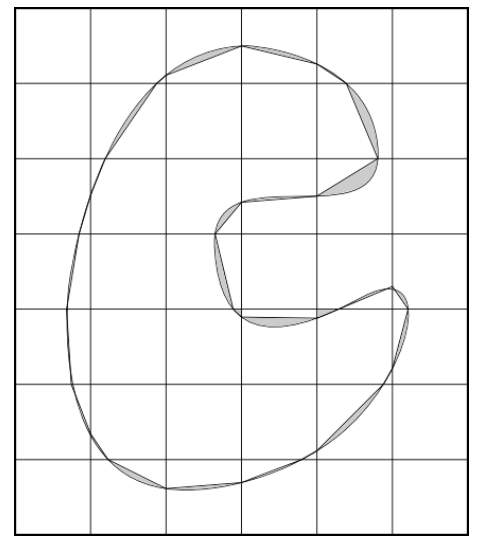

(a) Linha poligonal.

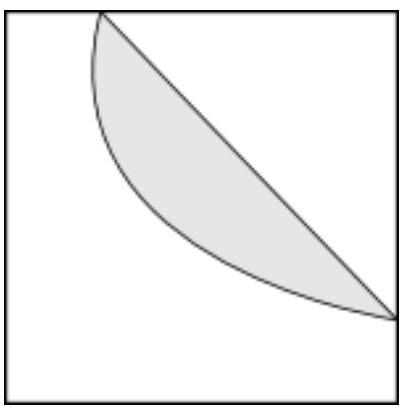

(b) Região entre um lado de $P$ e $f r(D)$

Fonte: $\mathrm{O}$ autor.

Esta nova partição produz uma partição $\mathcal{P}=\left\{t_{0}=a<t_{1}<\cdots<t_{n}=b\right\} \supset \mathcal{P}^{\prime}$ do intervalo $[a, b]$ obtida por meio da intersecção das linhas de grade gerada por $\mathcal{Q} \cup \mathcal{R}$ com $\operatorname{fr}(D)$. Ligando os pontos $\gamma\left(t_{i}\right)$ em sequência obtém-se uma nova linha poligonal $P$ que atende (4), isto é

$$
\left|\int_{\gamma} P d x+Q d y-\int_{P} P d x+Q d y\right|<\frac{\varepsilon}{2}
$$

de acordo com a observação 2 . Tal linha define uma região poligonal $\Pi$ contida em $V \cup D{ }^{7}$

A região $(D-\Pi) \cup(\Pi-D)$ (sombreada na Figura 6a) está contida nos retângulos que contêm pontos de $f r(D)$ (Figura 6b), logo

$$
\text { a. }((D-\Pi) \cup(\Pi-D))=\text { a. }(D-\Pi)+\text { a. }(\Pi-D)<\frac{\varepsilon}{2 M}
$$

Com isso, chamando $F(x, y)=\frac{\partial Q}{\partial x}-\frac{\partial P}{\partial y}$ temos:

$\log 0$

$$
\iint_{D} F(x, y) d x d y=\iint_{\Pi} F(x, y) d x d y+\iint_{D-\Pi} F(x, y) d x d y-\iint_{\Pi-D} F(x, y) d x d y
$$

$$
\begin{aligned}
\left|\iint_{D} F(x, y) d x d y-\iint_{\Pi} F(x, y) d x d y\right| & \leq\left|\iint_{D-\Pi} F(x, y) d x d y\right|+\left|\iint_{\Pi-D} F(x, y) d x d y\right| \\
& \leq M(a \cdot(D-\Pi)+a \cdot(\Pi-D)) \\
& <M \cdot \frac{\varepsilon}{2 M}=\frac{\varepsilon}{2}
\end{aligned}
$$

isto é

$$
\left|\iint_{D}\left(\frac{\partial Q}{\partial x}-\frac{\partial P}{\partial y}\right) d x d y-\iint_{\Pi}\left(\frac{\partial Q}{\partial x}-\frac{\partial P}{\partial y}\right) d x d y\right|<\frac{\varepsilon}{2}
$$

Diante das desigualdades (5) e (6) finalizamos a prova do teorema.

${ }^{7}$ A existência da região П é garantida pelo Teorema de Jordan: Se $\gamma$ é uma curva de Jordan, isto é, é simples e fechada, então $\mathbb{R}^{2}-\{\gamma\}$ possui duas componentes conexas, uma limitada e outra ilimitada, cuja fronteira comum é $\{\gamma\}$ (demonstração para o caso de $\gamma$ ser uma linha poligonal, bem como o caso geral, pode ser encontrada em Marinho (2005)). 


\section{Referências}

ACKER, F. Análise vetorial clássica. Rio de Janeiro: SBM, 2011.

COLLEY, S. J. Vector calculus. 4th ed. [S.1.]: Pearson Education, 2012.

COURANT, R. Differential and integral calculus. [S.1.]: Blackie \& Son Limited, 1936. v. 2.

GONÇALVES, M. B.; FLEMMING, D. M. Cálculo B. 2. ed. São Paulo: Pearson Prentice Hall, 2007.

GUERRA, M. Teoremas de Green e Stokes. Análise matemática IV, 2010. Disponivel em: https://www.passeidireto.com/arquivo/76343954/teoremas-de-green-e-stokes. Acesso em: 21 abr. 2020.

LANCELOTTI, S. Teoremi di Green, Stokes e Gauss. Lezioni di Analisi Matematica III, 201-? Disponivel em:

http://calvino.polito.it/ lancelotti/didattica/analisi2_new/approfondimenti/approfondimenti_gr een_stokes_gauss.pdf. Acesso em: 28 fev. 2019.

LEITHOLD, L. O cálculo com geometria analítica. 3. ed. São Paulo: Harbra, 1994. v. 2.

LIMA, E. L. Meu professor de matemática e outras histórias. Rio de Janeiro: SBM, 1991.

LIMA, E. L. Curso de análise. 11. ed. Rio de Janeiro: Impa, 2015. v. 2.

LIMA, E. L. Curso de análise. 14. ed. Rio de Janeiro: Impa, 2016. v. 1.

LINS NETO, A. Funções de uma variável complexa. Rio de Janeiro: Impa, 2008.

MARINHO, F. C. V. Teorema de separação de Jordan-Brower-Alexander. 2005. 127 f.

Dissertação (Mestrado em Ciências) - Universidade Federal do Rio de Janeiro, Rio de Janeiro, 2005.

MUNKRES, J. R. Analysis on manifolds. [S.1.]: Addison-Wesley Publishing Company, 1991.

PISKUNOV, N. Cálculo diferencial e integral. 11. ed. Porto: Lopes da Silva, 1997. v. 2.

SPIVAK, M. Calculus on manifolds. [S.1.]: Addison-Wesley, 1965.

STEWART, J. Cálculo. 6. ed. São Paulo: Cencarge Learning, 2009. v. 2.

THOMAS, G. B. et al. Cálculo. 11. ed. São Paulo: Addison Wesley, 2009. v. 2. 